\title{
Le Management Des Connaissances Au Niveau Des Etablissements D'enseignement Superieur : Cas Des Universites Publiques Marocaines
}

\author{
Abdelilah Elkharraz, $\mathrm{PH}$ \\ Ouail El Kharraz, PH \\ Khalid Chafik, PES \\ Equipe de Recherche: Management \& \\ Systèmes d'information (ER-MSI) \\ Ecole Nationale de Commerce et de Gestion de Tanger \\ Université Abdelmalek Essaâdi, Maroc
}

Doi: 10.19044/esj.2018.v14n4p350 URL:http://dx.doi.org/10.19044/esj.2018.v14n4p350

\begin{abstract}
This study aims to answer questions on the place, the role and the impact of the knowledge management to improve and develop the Moroccan university. The research is mainly based on an empirical study carried out on the implementation status of the knowledge management in eight Moroccan universities. The obtained answers show important results not only theoretically but also, and especially, empirically.

The conducted study is based on a qualitative approach characterized by a descriptive analysis of the current state by referring to the technique of semistructured interviews with different officials at the presidency level in these universities.

Despite the absence of a strategy formalised by the knowledge management within these universities, the analysis of the data collected shows that these practices have a positive effect on improving the quality of learning and, when making decisions, factors like the dominant culture, the socio-technical state and the decentralisation play a crucial role in adopting strategies based on the knowledge management.
\end{abstract}

Keywords: Knowledge Management, Moroccan university, Development of university

\section{Résumé}

Cette étude se propose de répondre à des questions portant sur la place, le rôle et l'impact du management des connaissances dans l'amélioration et le développement de l'université marocaine. La recherche repose 
essentiellement sur une étude empirique réalisée sur l'état de l'application du management des connaissances au sein de huit universités marocaines. Les réponses obtenues démontrent d'importants résultats non seulement sur le plan théorique mais aussi et surtout sur le plan empirique.

L'étude menée s'est basée sur une approche qualitative caractérisée par une analyse descriptive de l'existant en se référant à la technique d'entretien semi directif avec des différents responsables au niveau des présidences de ces universités.

L'analyse des données recueillies montre que malgré l'absence de stratégie formalisée du management des connaissances au sein de ces universités, ces pratiques ont un effet positif sur l'amélioration de la qualité de l'apprentissage au sein de celles-ci et que des facteurs comme la culture dominante, l'état sociotechnique et la décentralisation, quant à la prise de décision, jouent un rôle fondamental dans l'adoption de stratégies basées sur la gestion des connaissances.

Mots-clés: Management des connaissances, Université marocaine, Développement de l'université

\section{Introduction}

L'avenir repose sur notre aptitude à produire un système éducatif efficace. Si nous ne réussissons pas ce pari notre futur sera à coup sûr compromis.

Nul ne doute aujourd'hui que l'enseignement est le paramètre clé dans toute équation de développement. Les pays qui l'ont compris sont aujourd'hui ceux qui gouvernent le monde, alors que ceux qui tardent encore à l'admettre pataugent dans le sous-développement et la misère.

Ainsi, cet environnement difficile engage les universités à relever les défis et les chercheurs à analyser d'autres sphères d'investigation turbulentes et complexes. Cette complication provient aussi des limites des instruments et des approches du management traditionnel qui deviennent peu à peu incapables de répondre aux nouvelles exigences du marché et de la société et empêchent d'appliquer un pilotage transverse basé sur la connaissance et sur les nouveaux facteurs-clés du succès.

On passe alors progressivement, d'une approche centrée sur l'outil de production (bien ou service) à une approche de valorisation des savoirs (Malone, 2002). Dès lors, le management des connaissances peut être considéré aujourd'hui comme un enjeu majeur pour les universités : il permettra à ces dernières d'améliorer la qualité d'apprentissage de leurs étudiants et de s'orienter vers un management basé sur la flexibilité, l'innovation et la réactivité. 
De plus, les universités sont largement considérées non seulement comme des établissements d'enseignement, mais aussi comme des organisations qui créent, préservent et diffusent des connaissances et qui contribuent positivement au soutien social des communautés.

Par ailleurs, et en raison d'une forte concurrence au niveau de l'enseignement supérieur, un certain nombre d'universités ont mis en œuvre des stratégies et des outils qui améliorent les capacités des étudiants et la qualité des résultats de recherche et permettent de fournir plusieurs services communautaires afin de renforcer leur compétitivité (Oosterlinck, 2002).

Parmi ces stratégies et ces outils, le management des connaissances joue un rôle très important dans l'amélioration de l'efficience et l'efficacité de ces universités.

Et afin de mieux cerner notre sujet d'étude, nous le soumettrons à l'épreuve, à travers une recherche exploratoire de l'état des lieux et des conditions de développement des stratégies de management des connaissances, au sein des universités marocaines.

Pour une meilleure analyse de cette question, nous avons suivi une approche mixte regroupant à la fois étude intervention et étude empirique. Cette démarche est autant plus pertinente dans les problématiques de recherche peu explorées et/ou la littérature fait défaut comme dans le cas de notre étude. De plus, et dans le cadre de cette analyse, nous adopterons une approche système d'information, indissociable des aspects culturels et organisationnels du knowledge management.

Reste à signaler à ce niveau que notre réflexion portera sur le traitement et l'analyse des trois aspects suivants:

- Définitions et concepts clés.

- $\quad$ Contexte, problématiques et méthodologie de recherche.

- Résultats et recommandations.

\section{Definitions et concepts cles}

Avant de donner une définition claire du «management des connaissances ", il est essentiel, d'abord, à nos yeux, d'expliciter la notion du terme « connaissances ».

\section{Notion de « connaissance »}

En fait, et d'une manière générale on peut aborder la notion de connaissance selon cinq grandes dimensions (Earl, 2001) :

\section{Dimension hiérarchique}

Dans cette approche, une distinction nette est réalisée entre les notions de donnée, d'information et de connaissance. Une donnée est un élément brut, hors de tout contexte. Une information est une donnée mise en contexte. Et 
« une connaissance » est une information comprise, c'est à dire assimilée et utilisée par un individu permettant d'aboutir à une action.

\section{Dimension épistémologique}

Il s'agit, ici, de considérer la connaissance sous deux formes : explicite et tacite. Alors que «la connaissance explicite » est une «connaissance » facilement exprimable et transmissible, «la connaissance tacite » est fortement ancrée et indissociable des individus qui la détiennent.

\section{Dimension d'objet}

Dans cette dimension, « la connaissance » est considérée comme un élément que l'on peut stocker et manipuler. Le concept de mémoire organisationnelle qui vise à formaliser les connaissances de l'organisation s'inscrit dans cette perspective.

\section{Dimension processuelle}

La dimension processuelle se compose de deux perspectives. La première considère «la connaissance » comme une condition d'accès à l'information. Et la seconde comme une capacité à influencer des actions futures : savoir pour agir.

\section{Dimension des niveaux d'analyse et d'acteurs}

Il s'agit, ici, d'étudier « la connaissance » suivant différents niveaux d'analyse afin de distinguer : «la connaissance individuelle », « la connaissance de groupe », «la connaissance organisationnelle » et «la connaissance inter-organisationnelle ». Il s'agit également d'étudier les interactions sociales entre ces niveaux pour comprendre comment les connaissances individuelles deviennent des connaissances collectives.

Ainsi et d'après ce qui précède, « la connaissance » peut être définie comme la somme de ce qui est connu, le corps de la vérité, l'information et les principes acquis par l'humanité (Oosterlinck, 2002).

\section{Définition de « management des connaissances »}

En fait, plusieurs tentatives de définition existent déjà dans les manuels et les recherches portant sur le management des connaissances. Cependant, nous essayerons d'apporter notre propre contribution à ce domaine. Ainsi, on peut définir le management des connaissances pour les universités comme: « un management composé d'un ensemble de pratiques et d'approches visant à identifier, à créer, à utiliser, à capitaliser, à partager et à transférer les connaissances au sein des universités afin d'améliorer la qualité du produit final (Compétences de l'étudiant ou Nouvelle connaissance) ». 
De plus, il est vrai qu'il n'y a pas de méthode universelle pour appréhender le management des connaissances, mais il existe une multitude d'approches (El kharraz, 2013) à ce niveau :

- $\quad$ Le capital intellectuel mouvement : Avec Leif Edvinsson et Tom Stewart (Edvinsson, 1997) (Stewart, 1997): Ici, l'accent est mis sur la collaboration, y compris les concepts de planification sociale de la communauté de pratique, les processus de consultation, la participation du public et une gamme de technologies de collaboration. Une grande partie de ces travaux provient de la recherche par Etienne Wenger (Wenger, 1998) et le Lotus Institute (maintenant absorbé par IBM Research).

- L'approche culturelle : Il est difficile de classer toutes les organisations dans une seule approche culturelle. Mais, généralement les auteurs distinguent d'une part l'approche anglo-saxonne où le management des connaissances se situe en aval : "la connaissance » nécessaire pour satisfaire les différents besoins existe déjà au sein de l'organisation et peut faire l'objet d'une médiation par la technique. D'autre part, il y a l'approche japonaise centrée sur le partage de l'information, les prises de décisions partagées et la recherche de consensus où l'homme occupe une place primordiale qui précède celle de la technologie.

- Un ensemble de travaux dérivés de la théorie de l'information : Avec Larry Prusak et Thomas H. Davenport (Davenport \& Prusak, 1996): la théorie est liée à la conversion de connaissances tacites intégrées dans la codification des connaissances explicites, permettant le partage des connaissances avec succès comme cela a été souligné par Ikujiro Nonaka et Takeuchi Hirotaka (Nonaka \& Takeuchi,1995). C'est probablement la principale école de pensée, telle qu'elle est représentée par des publications et des développements ultérieurs par des auteurs comme Probst (Probst, 1998), Von Krough (Krough \& Roos, 1995) \& Malhotra (Malhotra, 2000).

- L'analyse stratégique : Pour Crozier et Freidberg (Crozier \& Freidberg, 1977), une organisation ne contraint jamais un acteur malgré ses prescriptions formelles. En principe, celui-ci garde toujours des marges de liberté et de négociation qui permettent de faire que sa participation soit payante. Et, à ce niveau, les deux auteurs ont déterminé quatre sources de pouvoir, à savoir : La maîtrise d'une compétence ou d'une expertise qui a une valeur pour l'organisation, la capacité d'édicter des règles et des procédures ou des cadres de fonctionnement, le contrôle des informations internes et la communication au sein de l'organisation.

- L'approche systémique : Les différents acteurs de l'organisation sont, en permanence, en interaction et l'approche systémique prône toujours la supériorité du groupe sur la somme des individus qui le forment, ainsi que la supériorité des connaissances mises en commun pour en créer de nouvelles. Ici, le retour d'expérience (ou de rétroaction) joue un rôle très important pour 
rétablir l'organisation dans son état initial ou de maintenir l'équilibre du système en question.

- $\quad$ L'organisation apprenante : Les personnes et les groupes doivent se préparer et s'adapter régulièrement à des situations inattendues : la création et la transformation des savoirs entre l'individu et le groupe, entre les connaissances tacites et les connaissances explicites, est mise en œuvre, en permanence, permettant ainsi le développement continu et adapté de la structure et des éléments qui la forment. Les membres de l'organisation deviennent non seulement des exécutants (pas même des acteurs), mais également des auteurs.

- L'organisation réseau : L'organisation en réseau permet d'établir une démarche efficace de management des connaissances. Car le réseau induit le partage, la mutualisation et facilite la formation et la création de connaissances: les réseaux d'experts ou les réseaux de compétences permettant une reconnaissance officielle des compétences collectives.

L'organisation des connaissances est un aspect très important du concept de "gestion des connaissances ». Ses fondements théoriques souffrent de la grande imprécision du vocabulaire utilisé pour en décrire les concepts et concevoir un système d'information dont la fonction est justement d'organiser des connaissances pour restituer un savoir à partir de faits observés et de données rassemblées (Beau, 2015).

\section{Contexte, Problematique Et Methodologie De Recherche}

Les approches présentées, ci-dessus, concernent l'organisation en général. Cependant, une question s'impose : Quels sont les principaux moteurs de changement dans la gestion de connaissances que connaissent les universités?

\section{Contexte de l'étude}

Il est nécessaire d'étudier le contexte à double niveau, mondial et national, afin de mieux comprendre l'importance de cette recherche.

\section{a. Contexte mondial :}

En fait, plusieurs éléments peuvent être évoqués à ce niveau :

- La mondialisation se traduit concrètement par une libre circulation des connaissances et des informations, ce qui amène les universités à s'ouvrir de plus en plus sur elles-mêmes et sur leur entourage (Abdussattar, Susan, 2001).

- Les économies mondiales convergent vers «l'économie du savoir» incorporant ainsi une part de plus en plus grande « d'immatériel ». Ceci pousse les universités à suivre la même tendance (Bruno, 2002).

- Le besoin de formation s'exprime de plus en plus, tout au long de la vie des individus et des organisations, nécessitant donc de la part des 
universités d'être capables de répondre à tout moment aux exigences des demandeurs (Pomian, Roche, 2002).

- Les évolutions technologiques rapides et les changements incessants au niveau du marché du travail impliquent de la part des universités l'adoption de politiques de Knowledge Management plus souples et plus réactives (Perrin, 2003).

- L'envie pressante des universités d'institutionnaliser l'informel à travers la reconnaissance, voir l'encouragement à la constitution de « réseaux d'échange de savoirs » ou « communautés de pratiques », la « formalisation » de procédures ou la constitution de bases de données, telles qu'elles sont promues par les théories de Knowledge Management (Vincent, Reza, 2004).

\section{b. Contexte national :}

A l'échelle nationale, cette étude coïncide avec un environnement qui se caractérise par (Lamrini, 2005) :

- L'engouement des différentes instances politiques marocaines envers l'amélioration de l'université marocaine. Et ce à travers des programmes qui visent la mise à niveau et l'extension des infrastructures, l'augmentation de la capacité d'accueil des universités, le renouvellement des équipements et l'amélioration de l'offre de l'enseignement supérieur, la promotion de la recherche scientifique et l'amélioration de la qualité des prestations des œuvres sociales en faveur des étudiants.

- La réforme pédagogique appliquée à grande échelle par le ministère de l'enseignement supérieur pour améliorer le niveau de nos universités.

- L'adoption au sein des universités marocaines d'une nouvelle méthodologie encouragée par le ministère visant à instaurer un apprentissage par compétences au lieu d'un apprentissage par mémorisation (modèle américain emprunté au monde de l'industrie).

- L'adoption par le Maroc de l'architecture européenne (LMD) au niveau de ses universités qui se base sur le système modulaire afin de faciliter la mobilité nationale et internationale des étudiants et d'améliorer le rendement de son système éducatif.

- Les changements au niveau du marché de travail marocain qui demande de plus en plus des diplômés compétents capables de combler les besoins spécifiques des entreprises marocaines.

En général, nous pouvons dire que l'université marocaine se doit aujourd'hui de relever trois défis essentiels :

$1^{\text {er }}$ défit : « La faiblesse du rendement interne et externe ».

$2^{\text {ème }}$ défit : « La capacité à répondre de façon effective aux besoins de développement économique de notre pays et en particulier les besoins qui sont exprimés à travers les programmes de développement sectoriel ». 
$3^{\text {ème }}$ défit : « La mondialisation de la formation universitaire qui fait que l'espace universitaire est aujourd'hui un véritable espace internationalisé »».

\section{Problématique de recherche}

Il s'agit à ce niveau de l'étude de la problématique du management de connaissances en général et surtout au niveau de l'université.

\section{a. Problématiques du management de connaissances :}

Signalons, tout d'abord, 1'existence de quatre grandes problématiques du management de connaissances (Duizabo, Guillaume, 1997) :

$>\quad$ La création des connaissances ;

$>\quad$ La capitalisation des connaissances ;

$>\quad$ Le transfert des connaissances ;

$>\quad$ Et le partage des connaissances.

* Création des connaissances :

Il est possible de déterminer quatre étapes pour créer des connaissances (Nonaka, 1991) :

- Socialisation : Il s'agit d'une phase où une personne transmet et partage directement ses connaissances tacites avec une autre personne. C'est le passage du tacite vers le tacite.

- Extériorisation : C'est une phase qui consiste à l'énonciation d'un fondement de connaissances tacites en connaissances explicites partageables par tous les membres. Il s'agit alors d'un passage du tacite à l'explicite.

- Combinaison : C'est le passage d'explicite à explicite où une personne peut introduire des parties de connaissances dans un nouvel ensemble.

- Intériorisation : Il s'agit de l'intégration des connaissances explicites. C'est le passage d'explicite à tacite.

\section{* Capitalisation des connaissances :}

Les buts poursuivis ici seront d'identifier, de localiser, de modéliser, de formaliser et d'archiver les connaissances.

Cependant, toutes les connaissances ne peuvent être gardées et préservées pour des raisons techniques et financières. Il faudra alors choisir celles qui représentent plus d'intérêt à être formalisées (Grundsten, 2000).

\section{* Transfert des connaissances :}

Pour transférer les connaissances au niveau des universités, la formation constitue un outil indispensable (Couraud, 2003). En fait, il existe plusieurs types de formations (formation classique en salle, formation par stage, etc.).

\section{* Partage des connaissances :}

Ici, on parle essentiellement de solutions technologiques (Groupware, Intranet, etc.) et d'approches stratégiques (Partenariats, Communautés de 
recherche, etc.) susceptibles d'aider l'université à diffuser et à recevoir l'ensemble des informations et des connaissances des autres acteurs (Charlot, Lancini, 2002).

\section{b. Le management de connaissances à l'Université :}

Ensuite, il est nécessaire de rappeler à ce niveau que le management des connaissances est devenu une priorité majeure pour le secteur privé comme pour le secteur public, pour le secteur industriel comme pour le secteur de service.

Toutes les institutions stockent, accèdent et diffusent des connaissances d'une manière ou d'une autre. La question est de savoir quelle est la valeur ajoutée aux produits et services qu'ils fournissent par l'utilisation efficace de ce capital de connaissances (Milam, 2001).

Par sa nature, l'environnement universitaire est approprié pour l'utilisation des principes et des méthodes de gestion des connaissances (Mikulecky, Mikulecka, 1999).

Les établissements d'enseignement supérieur ont d'importantes possibilités d'appliquer les pratiques de management des connaissances pour appuyer chaque partie de leur mission. Cette gestion des connaissances ne devrait pas être considérer par les établissements d'enseignement supérieur comme une idée radicalement nouvelle, mais plutôt comme une nouvelle orientation sur leur raison d'être (Kidwell, Jillinda, Vander, Karen, Sandra, 2000).

La figure suivante (Blaise, 2001) nous montre quelques aspects très intéressants de l'application du management de connaissance au niveau des universités :

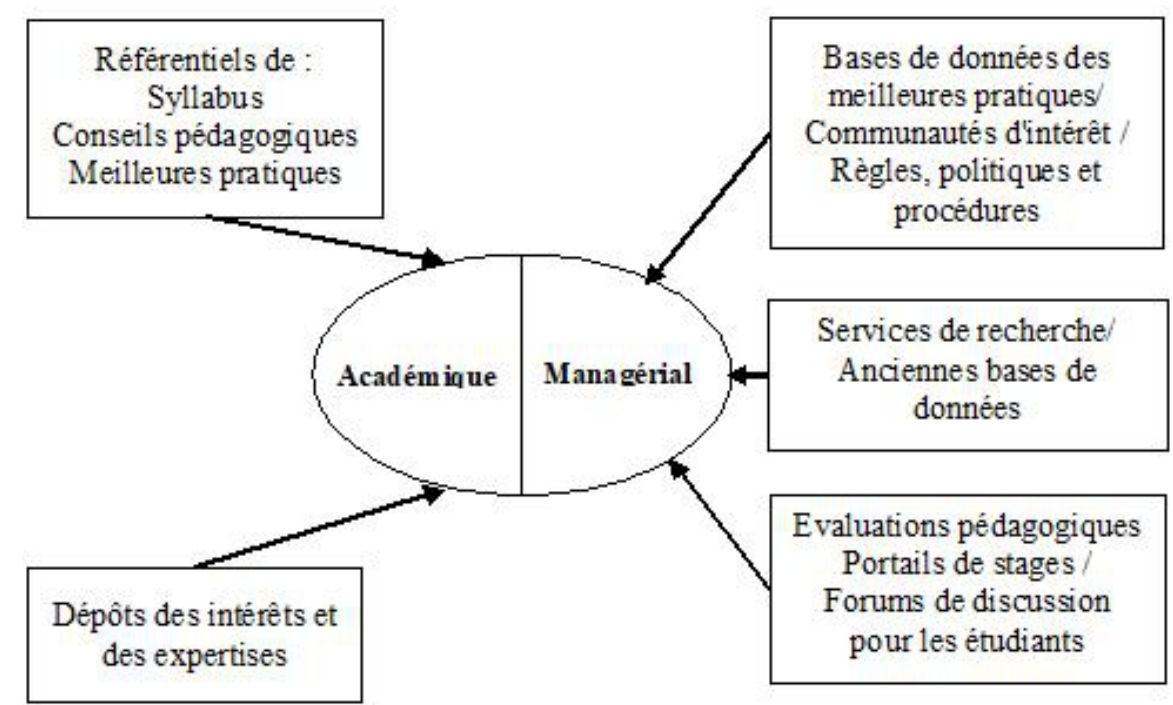

Figure 1 : Les types d'applications du management de connaissance à l'Université 
Ici, deux d'exemples concrets sont répertoriés sous «Universitaire»: l'un portant sur les questions relatives aux programmes et l'autre avec la recherche.

De l'autre côté et pour le « Managérial », le partage de l'expérience et des «meilleures pratiques» peut être atteint par:

(1) la mise en place de référentiels de programmes de cours, bénéfiques pour les étudiants et les professeurs;

(2) fournir des indications sur les évaluations des différents styles et pratiques pédagogiques;

(3) mettre en évidence les leçons tirées des expériences d'enseignement à distance; et

(4) créer des forums en ligne ou des communautés de pratique pour l'échange de connaissances tacites et d'expériences sur le terrain.

Reste à signaler à ce stade que la recherche menée a pour objectif de répondre à un ensemble de points qui s'intéressent au management des connaissances au niveau des universités marocaines mais surtout à la problématique suivante :

«Les stratégies de management de connaissances au niveau des universités marocaines : Etat des lieux et conditions de développent».

\section{Méthodologie de recherche}

Dans la réalisation de cette recherche, nous avons opté pour une approche mixte combinant étude empirique et étude de cas. Premièrement, l'étude empirique a touché huit universités marocaines dont une partie des résultats est présentée dans ce papier; deuxièmement, l'étude de cas fut consacrée aux stratégies de management des connaissances au sein de l'université Abdelmalek Essaadi, dont les résultats ont été présentés dans un autre article (El kharraz \& Chafik, 2012).

En outre, il convient de signaler que la légitimité de ce choix mixte repose sur le fait que les risques éventuels de défaillance d'une étude sont compensés par les forces de l'autre. Ainsi, le chercheur peut accorder plus de confiance à ses conclusions.

De plus, les informations recueillies, lors de la phase quantitative (Etude exploratoire), nous aideront à mieux appréhender les observations tirées de la réalité des universités marocaines. L'analyse des variables complexes, telles les pratiques informelles, ne pourrait être opérée sans un recours aux méthodes qualitatives (Etude clinique).

Par ailleurs, l'étude empirique sur les pratiques de management des connaissances a été réalisée sur la base d'un questionnaire guide rempli lors d'interviews directes au niveau de huit universités publiques marocaines. Le choix de l'enseignement supérieur public et non privé revient au fait que le défi majeur de l'université publique est lié à la massification de 
l'enseignement supérieur au Maroc. L'université publique absorbe presque la totalité de la demande de l'enseignement supérieur au Maroc.

Il reste à ajouter ici, que nous avons visé lors de notre étude empirique un ensemble de responsables chargés de la pédagogie, de la recherche et de la gouvernance administrative au niveau des présidences des différentes universités interviewées.

\section{Resultats Et Recommandations \\ Résultats de l'étude}

Les résultats de la recherche empirique sur le management des connaissances au sein des universités marocaines sont très importants.

\section{a. Les pratiques de management des connaissances au sein des universités marocaines :}

D'après les résultats, nous constatons que la moitié de ces universités (un peu plus que 50\%) dispose d'une politique de management des connaissances.

Aussi, concernant la communication au sein des universités marocaines, nous remarquons que malgré la forte existence de bases de données électroniques $(75 \%) \&$ de communication informelle $(75 \%)$ l'utilisation de la documentation écrite reste largement dominante (100\%).

Nous constatons, également, que la majorité des universités marocaines considère que les programmes enseignés répondent positivement au développement des capacités des étudiants (75\%) et aux besoins du marché de travail $(87,5 \%)$. Cependant, $87,5 \%$ de ces universités ne disposent pas de programmes permettant le suivi de la carrière de ses lauréats et même pas d'un responsable dédié à la gestion des connaissances.

Les résultats montrent aussi que toutes les universités marocaines disposent de contrats de partenariats et de collaboration en management des connaissances à l'intérieur et à l'extérieur du Maroc.

Nous notons, enfin, la présence d'intranet comme moyen de diffusion de connaissances et de communication dans la majorité des universités marocaines.

b. Niveau d'application du management des connaissances pour les universités marocaines :

Concernant cet élément, les résultats obtenus sont les suivants :

- $62,5 \%$ des universités marocaines considèrent que le niveau de création des connaissances au sein de ses entités est satisfaisant.

- $\quad$ La satisfaction sur le niveau de capitalisation, de transfert et de partage des connaissances est à hauteur de $87,5 \%$ au niveau des universités interrogées.

c. Impact de l'application du management des connaissances sur les universités marocaines : 
Les réponses enregistrées démontrent que les responsables des huit universités interrogées pensent que plus le niveau de l'application des stratégies de mangement des connaissances est grand, meilleure est la qualité de l'enseignement et de l'apprentissage.

Les résultats indiquent également que $87,5 \%$ des personnes questionnées au niveau des universités marocaines croient que plus la situation sociotechnique (infrastructures, lois et relations sociales) est stable, plus il y a amélioration de l'enseignement et de l'apprentissage.

\section{d. Management de connaissances : Autres résultats}

D'autres résultats importants se présentent comme suit :

$75 \%$ des universités marocaines appliquent la politique du ministère de l'enseignement supérieur concernant ce domaine dans leurs stratégies de management des connaissances.

- $\quad 62,5 \%$ des répondants croient que l'existence de différentes branches d'études et de différents responsables au sein des universités rend difficile la mise au point de pratiques communes de management des connaissances pour l'ensemble de ces dernières.

$62,5 \%$ des universités n'assurent pas une formation sur les différentes stratégies de management des connaissances pour ses responsables.

- $\quad 56 \%$ des universités exercent un contrôle sur les connaissances enseignées au niveau des différentes branches d'études.

- $\quad 50 \%$ des présidences des universités contrôlent les différentes méthodes pédagogiques de transfert de connaissances utilisées au sein de leurs universités.

- $\quad 43,5 \%$ des responsables des différentes composantes des universités marocaines ne disposent pas d'une liberté totale quant à l'application ou non des différentes stratégies de management des connaissances.

\section{Recommandations}

Pour le développement d'un management des connaissances de qualité au niveau de l'université marocaine, plusieurs recommandations peuvent être avancées, cependant, les plus indispensables, à nos yeux, se présentent comme suit :

- L'université marocaine devrait établir un cahier de missions pour :

1. Expliquer clairement les différentes fonctions ;

2. Déterminer les responsabilités ;

3. Fixer les résultats espérés ;

4. Etablir les budgets alloués pour la réussite de chaque mission etc.

Ce document devrait être discuté à différents niveaux de l'université, notamment par les intervenants qui occupent des postes de responsabilité pouvant influer sur la prise de décision en matière de management des connaissances. 
- Adopter une approche interdisciplinaire. Ainsi, la traditionnelle division entre les facultés, les écoles et au niveau des autorités pourrait être remise en question et par là même subir une évolution en profondeur.

- $\quad$ Comprendre que l'achat d'un très grand nombre d'ordinateurs n'est pas synonyme d'un meilleur système de management des connaissances.

- $\quad$ Préparer nos étudiants, autant que possible, à être les acteurs de demain d'une société de connaissances.

- $\quad$ Effectuer une évaluation ouverte et approfondie à la fin de chaque année au niveau de l'université. Et, si les résultats de celle-ci étaient en-deçà des attentes, l'université devrait être en mesure de développer un climat favorable à admettre même les répercussions les plus désagréables.

- Les responsables des universités devraient cesser d'adopter une approche administrative dans l'exécution de leurs tâches et opter, plutôt, pour une approche académique.

Reste à signaler ici, que l'application de ces recommandations ne constitue qu'une étape dans le processus de réussite d'un management des connaissances de qualité au sein de nos universités. Ces dernières sont appelées à jouer, dans une économie basée sur l'acquisition du savoir, un rôle de premier plan : celui de protagonistes pour fournir à la société un avantage concurrentiel au niveau national et international.

\section{Conclusion}

L'approche basée sur «la connaissance» est sans aucun doute l'événement sociologique le plus important de ces dernières années.

Dorénavant, nous vivrons un nouveau mode de management où « la connaissance » est une richesse à préserver et à manager. Son impact sur les stratégies managériales des organisations ne peut être négligé (Rowley, 2000).

La nature et le rôle joué par l'université la met normalement dans une situation favorable pour l'application réussie d'un management de connaissances de qualité.

Cependant, les résultats montrent que les universités marocaines ne sont qu'au début du parcours dans ce domaine.

Les origines de ce manque peuvent être recherchées, en premier lieu, dans la nature de l'environnement dans lequel ces universités ont évolué et, en deuxième lieu, dans la culture qui véhicule dans ces entités.

Ce qu'il faut, en fait, c'est la réalisation d'un processus de sensibilisation et de motivation qui aidera les responsables à mûrir, pour finalement permettre une prise de conscience complète vis-à-vis de l'application du management des connaissances.

Les nouvelles générations devraient être formées à ces pratiques; les anciennes devraient, peu à peu, s'y adapter. Cet effort se traduira, 
progressivement, par un changement de mentalité qui, à la longue, pèsera et fera évoluer les organisations.

Ceci dit, il est, à notre avis, très important de souligner que les universités continueront à jouer un rôle primordial dans la société de demain en supposant qu'elles sont prêtes à assumer leurs responsabilités considérables.

En guise de conclusion, nous dirons que l'étude menée ne constitue qu'une première étape pour la compréhension de la place et du rôle joué par le management des connaissances dans les universités marocaines et que d'autres études devraient être réalisées dans ce domaine et surtout en matière de comparaison entre les universités marocaines et les universités qui sont leader en management de connaissances. Ce qui facilitera la détection des erreurs et les manques à satisfaire.

\section{References:}

1. Abdussattar C., Susan E-H. 2001. Perspectives on education for knowledge management. 67th IFLA Council \& General Conference (16-25 août 2001). Boston. É.-U.

2. Beau F. 2015. «L'organisation des connaissances au cœur du système d'information ». Revue internationale des sciences commerciales, Communication \& management, Vol. 12, $\mathrm{N}^{\circ} 1$. pp. 1330.

3. Blaise C. 2001. « Knowledge management, organizational culture and Anglo-American higher education ». Journal of Information Science, 27 (3). pp. 129-137.

4. Bruno V. 2002. «Gérer des compétences et manager des connaissances ». Management et Conjoncture Sociale. N616 (automne 2002).

5. Charlot J-M., Lancini A. 2002. « De la connaissance aux systèmes d'information supports ». Coordonné par Rowe F. Faire de la recherche en systèmes d'information. Editions Vuibert. pp. 139-154.

6. Couraud G-E. 2003. « Le management de la formation au service de la gestion des connaissances » DESS Gestion des Ressources Humaines en Apprentissage, IAE de Strasbourg. p. 96.

7. Crozier M. \& Friedberg E. (1977), L'Acteur et le Système, Editions du Seuil. Paris.

8. Davenport T. H., Eccles R. G. and Prusak L. (1996). "Information Politics", MIT Sloan Management Review, 34, no. 1 (fall 1992): 5365.

9. Duizabo S. et Guillaume N. 1997. « Les Problématiques de Gestion des Connaissances dans les Entreprises ». Cahier $n^{\circ} 252$. Centre DMSP. Université Paris Dauphines (Février 1997). 
10. Earl M. 2001. « Knowledge management strategies : toward a taxonomy ». Journal of Management Information Systems, Volume 18, N¹.pp. 215-233.

11. Edvinsson Leif, 1997. "Developing intellectual capital at Skandia", Long Range Planning, Vol. 30, Issue 3, pp. 366-373. (June 1997).

12. El kharraz O. 2013. «Les pratiques des stratégies de management des connaissances au sein des universités marocaines $»$. Thèse de doctorat, ENCG de Tanger. p 18.

13. El kharraz O. et Chafik K. Juin 2012; "Les pratiques du management des connaissances au sein des universités marocaines : Etat des lieux et conditions de développement - Cas de l'université Abdelmalek Essaâdi"; Revue : "Dossiers de Recherches en Economie et Gestion" ; Numéro 1, pp. 80-101.

14. Grundsten Michel. 2000. «Repérer et mettre en valeur les connaissances cruciales pour l'entreprise ». 10ème Congrès International de 1'Association Française pour 1'Analyse de la Valeur (7-8 novembre 2000). Paris. France.

15. Jillinda J-K., Karen M-V., Sandra L-J. 2000. «Applying Corporate Knowledge Management Practices in Higher Education » ; Educause Quarterly. Volume 4. pp. 28-33.

16. Krogh, G. V. \& Roos, J. (1995). "Organizational Epistemology”. London: Sage Publications.

17. Lamrini A. 2005. Systèmes éducatifs, savoirs, technologies et innovation. Rapport thématique. $105 \mathrm{p}$.

18. Malhotra Y. (2000), Knowledge management and virtual organizations. Editeur Yogesh Malhotra.

19. Malone D. 2002. Knowledge management: A model for organisational learning. International Journal of Accounting Information Systems. Volume 3. Août 2002. pp. 111-123.

20. Mikulecky, P., Mikulecka, J. 1999. Active Tools for Better Knowledge Dissemination. ASIS 1999 Annual Meeting (29 Octobre - 04 Novembre). Washington D.C. É.-U.

21. Milam J-H. (Date de consultation: 01 Février 2017). Knowledge Management for Higher Education. ERIC Clearinghouse on Higher Education.

[en ligne].

URL: https://eric.ed.gov/?q=Knowledge+Management+for+Higher+Educat ion+\&id=ED464520

22. Nonaka I. and Takeuchi H. (1995), The Knowledge-Creating Company. How Japanese Companies Create the Dynamics of Innovation, Oxford University Press, New York

23. Oosterlinck A. (2002), (Date de consultation: 10 Février 2017). Knowledge Management in Post-Secondary Education : Universities. 
Organisation for Economic Co-operation and Development. [en ligne]. URL : www.oecd.org/edu/research/2074921.pdf

24. Perrin A. 2003. La valorisation du management des connaissances dans les organisations, mémoire de DEA, université de Nice sophia antipolis.

25. Pomian J., Roche C. 2002. Connaissance Capital: Management des connaissances et organisation du travail. Les éditions Sapientia \& Les éditions de l'Harmattan. $648 \mathrm{p}$.

26. Probst G FB (1998). "Practical Knowledge Management: a model that works". Prism.

27. Rowley J. 2000. Is Higher Education Ready for Knowledge Management?. The International Journal of Educational Management, 14, 7. pp. 325-333.

28. Stewart T.A. (1997). Intellectual Capital: The New Wealth of Organizations. Nicholas Brealey Publishing, London.

29. Vincent M-R., Reza K. 2004. Integrating Total Quality Management and Knowledge Management. Journal of Management Systems, Vol. XVI, No. 1. pp. 39-54.

30. Wenger E. (1998). Communities of Practice: Learning, Meaning, and Identity, Cambridge University Press. 